\title{
Germanica
}

Anderswo zwischen den Texten. Intertextualität als Illustration in Hugo von Hofmannsthals Das Bergwerk zu Falun

L'ailleurs à travers les textes. Intertextualité dans La Mine de Falun de Hugo von Hofmannsthal

The elsewhere through the texts. Inter-textuality in Hugo von Hofmannsthal's The Mines of Falun

\section{Thomas Eicher}

\section{OpenEdition}

\section{Journals}

Édition électronique

URL : http://journals.openedition.org/germanica/251

DOI : 10.4000/germanica.251

ISSN : 2107-0784

Éditeur

Université de Lille

Édition imprimée

Date de publication : 1 juin 2007

Pagination : 157-174

ISBN : 2-913857-19-1

ISSN : 0984-2632

\section{Référence électronique}

Thomas Eicher, «Anderswo zwischen den Texten. Intertextualität als Illustration in Hugo von Hofmannsthals Das Bergwerk zu Falun », Germanica [Online], 40 | 2007, Online erschienen am: 16 Februar 2010, abgerufen am 06 Oktober 2020. URL : http://journals.openedition.org/germanica/251 ; DOl : https://doi.org/10.4000/germanica.251

Ce document a été généré automatiquement le 6 octobre 2020.

(c) Tous droits réservés 


\title{
Anderswo zwischen den Texten. Intertextualität als Illustration in Hugo von Hofmannsthals Das Bergwerk zu Falun
}

\author{
L'ailleurs à travers les textes. Intertextualité dans La Mine de Falun de Hugo \\ von Hofmannsthal \\ The elsewhere through the texts. Inter-textuality in Hugo von Hofmannsthal's \\ The Mines of Falun
}

Thomas Eicher

\section{Vorbemerkung}

1 Als plurimedialer Text hat das Drama Seiten, die der Text, der einer Inszenierung zugrunde liegt, nicht in Gänze abbilden kann. Bühnenbild und Kostüme verleihen ihm Ambiente und Atmosphäre, ebenso wie Gestalt, Bewegung und Artikulation der Schauspieler - um nur einige Faktoren zu nennen ${ }^{1}$. Es sei aber im folgenden eine Konzentration auf den Dramentext gestattet und der Versuch gemacht, weitere mediale Komponenten auszusparen, so, als wäre der Dramentext lesbares Produkt eines Schreibprozesses, der sich wiederum aus einer Fülle von vorgängigen Texten speist.

Gerade im Falle des Hofmannsthalschen Textes kann man mit Fug von einem Prozeß sprechen. Seine Entstehungs- und Publikationsgeschichte zieht sich fast über ein halbes Jahrhundert hin. Der erste Akt erschien bereits 1900 im zweiten Band der Insel. Die anderen Akte wurden verstreut veröffentlicht, wobei die numerische Reihenfolge offenbar nicht der Entstehungschronologie entspricht. Schon der dritte Akt erschien erst postum 1932. Als Ganzes erfolgte ein Abdruck des Dramas erst 1946 in den Gesammelten Werken ${ }^{2}$.Trotz dieser Editionsgeschichte, die die Geschlossenheit des Werks 
unbeglaubigt läßt, möchte ich den Text doch als kohärentes Ganzes begreifen, das sowohl der Lektüre als auch der Bühnenaufführung standhält.

Der Dramentext hat seine liebe Last mit der Darstellung von Innenwelten: Was die handelnden Figuren denken, fühlen, imaginieren, empfinden, müssen sie - sofern die Regieanweisungen keine Kommentare dazu abgeben - eben äußern: mit Körper und Stimme. So bringen die Akteure bisweilen zum Ausdruck, was unsagbar ist. Gesprochene Worte sind scheinbar das nächstliegende Mittel, innere Befindlichkeiten kommunikabel zu machen: Jemand sagt, wie er sich fühlt, berichtet, was er geträumt hat - gesprächsweise oder im Monolog. Dadurch wird die Innenwelt zum hörbaren, lesbaren Text, den Ordnungsgefügen der Sprache unterworfen. Scheinbar von innen kommend, generiert sich in diesem Sprechakt ein ganzes Geflecht von Versatzstücken anderer Textwelten. Das Anderswo im Inneren des Sprechers bedarf der 'Bebilderung' durch das Anderswo zitierter und alludierter Texte. Doch beginnt die Intertextualität des Dramas - wie die Handlungsweise der Figuren - bereits im Nebentext. Titel, Personennamen und Regieanweisungen geben plakativ Auskunft über den Anspielungshorizont, vor dem sich die Innenwelten entfalten.

Hugo von Hofmannsthals Das Bergwerk zu Falun hat seine stofflichen Wurzeln in der Romantik. Der Titel stellt einen Bezug zu E.T.A. Hoffmanns Die Bergwerke zu Falun her, einer Geschichte aus den Serapionsbrüdern. Daß diese Erzählung Hoffmanns in einer mythischen Tradition wurzelt, die eine ganze Reihe romantischer Autoren zu literarischen Produktionen animieren konnte, verleiht dem Drama Hofmannsthals eine ungewöhnlich weit verästelte Tiefenstruktur. Das Anderswo dieses Textes hat drei Dimensionen:

1) Das Drama spielt in Schweden, zunächst in Göteborg, dann in Falun. Diese geographische Verortung ist jedoch so vordergründig wie beliebig. Sie erhält, wie ich zeigen werde, vor allem als Allusion funktionale Bedeutung.

2) Der Text entwickelt mit der Hauptfigur eine - auch im Wortsinne untergründige Figurenpsyche, die auf charakteristische Weise mit den räumlichen Gegebenheiten zwischen Unten und Oben, unter Tage und über der Erde, parallelisiert wird. Die Darstellung dieser konzeptuellen Opposition kommt obendrein nicht ohne Phantastik aus, die ihrerseits die innere Agonie des Helden bühnenwirksam in Szene setzt.

3) Wichtiger noch für die vielschichtige Semantik des Dramas ist seine Intertextualität, die die erwähnten Dimensionen erst zur vollen Entfaltung bringt. Durch diese Verweisstruktur schafft sich der Text einen gleichsam illustrativen Horizont. Darum ist es besonders wichtig, die Bedeutungsebenen, die sich aus den Prätexten speisen, zu rekonstruieren - zunächst natürlich mit Blick auf den Falun-Stoff, im weiteren Verlauf auch unter Zuhilfenahme anderer Quellen Hofmannsthals aus der Erzählliteratur der Romantik.

\section{Die Sage vom Bergwerk zu Falun}

5 Schon 1670 war in der Faluner Mardskin- (also Marderfell-)Grube knapp 150 Meter unter der Erde ein Bergmann namens Mathias Israelsson, genannt Fet-Mats, verschüttet worden. Seinen Leichnam fand man 1719, also 49 Jahre später, bei Grubenarbeiten unversehrt und wohlbehalten wieder. Er konnte dadurch von einem ehemaligen Arbeitskollegen identifiziert werden. Auch die inzwischen greise Verlobte des Toten konnte seine Identität bestätigen. Den durch Eisenvitriol konservierten 
Leichnam stellte man im Museum zur Schau. Doch der Glaskasten, in dem die sterblichen Überreste von Fet-Mats aufbewahrt wurden, konnte sie nicht mehr vor dem Zerfall schützen wie ehedem die Natur unter Tage. Als die Leiche nach 30 Jahren über der Erde unansehnlich wurde, begrub man sie endgültig auf dem Kirchhof von Stora Kopparberg ${ }^{3}$.

6 Der Fund im Jahre 1719 war zwar spektakulär, erlangte aber zu seiner Zeit über Skandinavien hinaus kaum Bedeutung. Der deutschen Romantik blieb es vorbehalten, diesen Stoff wiederzuentdecken. In Deutschland erschien er zuerst in einem wissenschaftlichen Kontext, in den naturphilosophischen Vorlesungen Gotthilf Heinrich Schuberts, wurde aber schon wenig später durch die Zeitschrift Jason populär gemacht. Sie druckte im Aprilheft des Jahres 1809 einen Auszug aus Schuberts Ansichten von der Nachtseite der Naturwissenschaft ab, versehen mit der Überschrift «Dichteraufgabe»" Im Gefolge dieser «Dichteraufgabe» erschien eine Vielzahl an Erzähltexten, später auch einige dramatische Dichtungen, zum vorgegebenen Thema ${ }^{5}$. Die Kalendergeschichte Johann Peter Hebels mit dem schönen Titel Unverhofftes Wiedersehen von 1811 dürfte am meisten zur Verbreitung des Stoffes beigetragen haben. Hebel verlegt das Auffinden des Leichnams ins 19. Jahrhundert, indem er es auf berühmt gewordene Weise mit dem Zeitgeschehen verbindet, das den Lesern seines Volkskalenders präsent ist: Krieg und Tod weisen hier auf die Begrenztheit menschlichen Handelns hin, auf einen fatalen Kreislauf, der in seiner Unausweichlichkeit dem Zyklus der Feldarbeit entspricht ${ }^{6}$.

7 Hebel wie Schubert nutzen die für deutsche Leser exotische Örtlichkeit Falun als Exempel. Doch es geht ihnen weniger um das geographische Anderswo als um das Ereignis selbst und seine metaphysische Dimension, die je unterschiedlich als Allmacht der Natur bzw. eines christlichen Gottes dargestellt wird. Einige Balladendichtungen im Gefolge der Jason-Publikation gehen da noch einen Schritt weiter, indem sie den Ort der Handlung nicht benennen (beispielsweise die Gedichte Arnims, Rückerts, Langbeins oder Pfizers) oder sogar verlegen, so z.B. Johann Nepomuk Vogl, der ein Salz(!)bergwerk einstürzen läßt.

8 In den Berichten und literarischen Texten über den Fund in Falun treffen sich verschiedene Motive der traditionellen Bergmannssagen. Gerhard Heilfurth hat die Erzählung Hebels in seiner Quellensammlung über Bergbau und Bergmann in der deutschsprachigen Sagenüberlieferung Mitteleuropas unter der Kapitelüberschrift «Wunderbare Rettung und Erhaltung» eingeordnet ${ }^{7}$. Dort steht jedoch eindeutig die Rettung im Vordergrund: Bergleute, die verschüttet viele Tage, Monate oder gar mehrere Jahre überleben können, weil ihnen wunderbare Hilfe zuteil wird. Daß jedoch eine Leiche nach vielen Jahren unversehrt geborgen wird, erscheint weit weniger ungewöhnlich als eine über die Maßen lange Schicht. Zum Sagenstoff können solche Funde vor allem deshalb werden, weil man sich in früheren Zeiten ihre Konservierung, die als Gegensatz zur gängigen Naturerfahrung erlebt wurde, nicht zu erklären wußte.

Die Sage aus Falun hat indessen noch mehr zu bieten als die im ungewissen Anderswo der Metaphysik verortete Urheberschaft einer überraschenden Wiederkehr. Sie verbindet den rätselhaften Fund mit dem Motiv der treuen Braut, das ebenfalls in den Bergmannssagen mehrfach nachgewiesen ist. Auf diese Weise entsteht eine literaturgeschichtlich besonders erfolgreiche Kombination. Das Erfolgsrezept des Stoffes ist indessen wohl kaum seine Authentizität. Eher wird man nach der literarischen Qualität seiner Verarbeitungen fragen müssen; und die ist bereits bei 
Schubert und Hebel in besonderer Weise ausgeprägt. Karlheinz Stierle hat in seiner Analyse des Unverhofften Wiedersehens herausgearbeitet, daß die Wirkung der Kalendergeschichte unter anderem durch die pointierte Handhabung der konzeptuellen Opposition von Leben und Tod erzielt wird, die bei Hebel in ein zweifach paradoxales Verhältnis gesetzt ist. Die alte Frau erscheint als lebendig-tot, die Leiche des Jünglings als tot-lebendig. Darüber hinaus bezeichnen diese Begriffspaare auch den Ablauf der Geschehensmomente in der Geschichte: Während am Anfang der Tod in die Lebensplanung des Paares eingreift, verspricht das Ende durch den Glauben der Alten an ein Leben nach dem Tod die Umkehrung der natürlichen Abfolge lebendig $\rightarrow$ tot $^{8}$. Die gut erhaltene Leiche stellt dabei symbolisch die Vergänglichkeit alles Organischen in Frage. Diese Deutung legt Mutmaßungen über die transzendentale Urheberschaft der Konservierung nahe, die eben jenen Einfluß des Wunderbaren darstellt, der das «Wesen» der Sagendichtung mitbestimmt. Schon bei Schubert wird eine symbolische Gegenüberstellung von lebendig-tot und tot-lebendig angelegt, bei Hebel kommt sie jedoch in Verbindung mit der Jenseitshoffnung der alten Frau zur eigentlichen Entfaltung. Dennoch bleibt auch hier ein sagentypisches Auftreten und Agieren des Wunderbaren aus. E.T.A. Hoffmann bereitet es mit seiner Weiterentwicklung des Stoffes vor. Ernst bei Hofmannsthal wird es inszeniert.

\section{Hoffmann zwischen Realien und Märchen}

10 E.T.A.Hoffmanns Die Bergwerke $z \mathfrak{u}$ Falun erweitert eine Variante der Schubert entnommenen «Dichteraufgabe» in signifikanter Weise um die Lebensgeschichte des am Ende verschütteten Bergmanns, der vom Erzähler erstmals mit dem in der Folgezeit auch durch andere Autoren aufgegriffenen Namen Elis Fröbom versehen wird. Was aber Hoffmanns Gestaltung des Falun-Stoffes, insbesondere seine Erweiterung, auszeichnet, ist die - nun nicht mehr sagen- sondern vielmehr märchenhaft anmutende - Begegnung des Bergmanns mit der Übernatur, in deren Dienst er sich zunächst stellt, sich aber dann nicht zwischen ihrer dämonischen Anziehungskraft und dem irdischen Liebreiz der Ulla Dahlsjö entscheiden kann, mit der er sich kurz vor dem - in gewisser Hinsicht erlösenden - Grubenunglück verlobt hat. Neben das geographische und historische Anderswo der Sage tritt also hier verstärkt ein phantastisches Anderwo.

11 Sah man bei Schubert und bei Hebel noch die ordnende Hand der Gottheit im Spiel, die die Überwindung des Todes in der Natur sinnfällig werden läßt, so halten bei E.T.A. Hoffmann die Dämonen Einzug. Mit der Bergkönigin und ihrem Sendboten Torbern wirken nun Kräfte, die das Gegensatzpaar Leben vs. Tod zeitweilig verschmelzen. Torbern, der Untote von Gnaden der Bergkönigin, geistert sowohl in der kollektiven Imagination der Bergleute als auch in der von Träumen reich bebilderten Vorstellungswelt des Elis Fröbom herum. Die Sage vom alten Torbern präfiguriert in mancherlei Hinsicht das Schiksal Elis'; denn auch Torbern wird am Johannistag verschüttet, nachdem er, aufgrund seiner enormen Kenntnisse verdächtigt, «mit der geheimen Macht, die im Schoß der Erde waltet und die Metalle kocht, im Bunde $»^{9} \mathrm{zu}$ stehen, vergeblich vor der Gewinnsucht gewarnt hat, mit der der Bergbau vorangetrieben wird. Doch Torbern ist für die Bergleute ein guter Geist, der lediglich für das Individualschicksal Elis' zum Verhängnis wird, indem er, wie die Mutter Erde und Bergkönigin, für den Jüngling sowohl Verheißung als auch Bedrohung bedeutet. 
12 Es zieht den Bergmann Elis schließlich in den Bereich zwischen Leben und Tod, einen Bereich, der ihm persönlich ein gleichsam höheres Leben verspricht. In der Außenperspektive hat er sich damit als Todgeweihter vom Leben verabschiedet. Wahnsinnig wird Elis Fröbom jedoch nicht, weil er vom stigmatisierten Besitzstreben ergriffen wird, sondern, weil es ihm weder erlaubt ist, sich über das Inzestverbot hinwegzusetzen und sich mit der 'Mutter' Erde $\mathrm{zu}$ vereinigen, noch Teil der menschlichen Gemeinschaft über der Erde $\mathrm{zu}$ werden ${ }^{10}$. Dabei ist es gerade das Sozialleben der Bergleute, das in den Bergwerken zu Falun als ernster, treuherziger und gemütvoller Umgang miteinander in einen wohligen Kontrast zum wilden Seefahrerleben gesetzt wird.

Der Fund der Leiche gerät bei E.T.A.Hoffmann zum matten Nachklang einer Geschichte, die eigentlich mit der Verschüttung ihr Ende bereits gefunden hat. Schon der Umfang dieses Appendix - in Relation zu dem der erzählten Lebensgeschichte des Elis Fröbom - läßt nicht im mindesten eine etwa der Kalendergeschichte Hebels vergleichbare Emphase aufkommen. So vermag auch das finale tot-lebendig nach dem Fund der Leiche in der Erzählung Hoffmanns nicht das lebendig-tot der Vorgeschichte des Helden zu neutralisieren. Im Gegensatz zur Hebelschen Version des Stoffes, wo die Braut im Weggehen ihrer Jenseitshoffnung Ausdruck verleiht, stirbt die alte Frau bei Hoffmann in der Umarmung mit dem Toten. Die Leiche des Elis Fröbom zerfällt bei dieser Umarmung zu Staub. Kein noch so trügerischer Anschein des Lebendigen bleibt bestehen. - Staub zu Staub, lautet die nihilistisch anmutende Botschaft der Hoffmannschen Version. Innerhalb des konzeptuellen Gefüges der Geschichte spielt auch das Bergwerk von Falun als magischer Ort der Sage keine signifikante Rolle mehr. Es wird zur Kulisse degradiert, zu einer Projektionsfläche der inneren Agonie des Helden. Für das moderne Erzählen erhält das Numinose seine volle Bedeutung erst im Innenraum der Psyche, die sich an ihm abarbeitet. Das spektakuläre Wiedersehen mit der konservierten Leiche wird unter diesen Umständen immer funktionsloser.

E.T.A.Hoffmann folgt in seiner Erzählung seinem Prinzip der Duplizität, das der Wirklichkeit ein im Inneren Geschautes gegenüberstellt ${ }^{11}$. In den Bergwerken erweist er sich weniger als «Gespenster-Hoffmann» denn als Kenner der Realia und als gebildeter Rezipient zeitgenössischer Diskurse aus Bergbau- und Reiseliteratur ${ }^{12}$. Er staffiert die Geschichte derart mit bergmännischen Fachbegriffen und landeskundlichen Details aus, daß sich auf der Ebene der Rahmenhandlung die Serapionsbrüder über diese Geschwätzigkeit des Erzählers Theodor lustig machen:

Aber gut ist es, lieber Theodor, daß du uns die Geschichte vorlasest, die wir alle, mein ich, etwas von der Bergmannswissenschaft, so wie von den Bergwerken von Falun und den schwedischen Sitten und Gebräuchen gehört haben. Andere würden dir mit Recht vorwerfen, daß du durch zu viele bergmännische Ausdrücke oft unverständlich wurdest, und manche würden sogar, da du so oft von dem schönen Öl sprichst, womit sich die Leute traktieren, auf den Gedanken geraten, daß die guten Faluner und Götaborger schnödes Baumöl saufen, da jenes Öl doch nichts anderes ist als ein schönes, starkes Bier ${ }^{13}$.

Damit wird nicht nur halb ironisch eine Worterklärung nachgeliefert und ein Hinweis auf jene Quellen gegeben, die Hoffmann zugänglich gewesen sein müssen, sondern auch über die explizit gemachten Voraussetzungen der Rezeption dieses Textes die Popularität der genannten Diskurse unter der zeitgenössischen Leserschaft angedeutet. Der konservierte Bergmann von Falun ist dem gebildeten Publikum bekannt, und die Ausgestaltung seines Lebensraums durch Versatzstücke der Schwedenreiseliteratur, 
die bis hin zu einem längeren wörtlichen Zitat aus Hausmanns Beschreibung der großen Pinge reicht, ist naheliegend. Auch über den Beruf des Bergmanns wird in der zeitgenössischen Literatur Einschlägiges verbreitet. Novalis etwa formuliert in Heinrich von Ofterdingen Wegweisendes zum Berufsethos der Bergleute :

Arm wird der Bergmann geboren, und arm gehet er wieder dahin. Er begnügt sich $\mathrm{zu}$ wissen, wo die metallischen Mächte gefunden werden, und sie $\mathrm{zu}$ Tage $\mathrm{zu}$ fördern; aber ihr blendender Glanz vermag nichts über sein lautres Herz. Unentzündet von gefährlichem Wahnsinn, freut er sich mehr über ihre wunderlichen Bildungen, und die Seltsamkeiten ihrer Herkunft und ihrer Wohnungen, als über ihren alles verheißenden Besitz. Sie haben für ihn keinen Reiz mehr, wenn sie Waaren geworden sind. [...] Mühseligkeiten erhalten sein Herz frisch und seinen Sinn wacker; er genießt seinen kärglichen Lohn mit inniglichem Danke, und steigt jeden Tag mit verjüngter Lebensfreude aus den dunkeln Grüften seines Berufs. [...] Sein einsames Geschäft sondert ihn vom Tage und dem Umgange mit Menschen einen großen Teil seines Lebens ab. Er gewöhnt sich nicht zu einer stumpfen Gleichgültigkeit gegen diese überirdischen tiefsinnigen Dinge und behält die kindliche Stimmung, in der ihm alles mit seinem eigenthümlichsten Geiste und in seiner ursprünglichen bunten Wunderbarkeit erscheint. [...] Wie ruhig arbeitet [...] der arme genügsame Bergmann in seinen tiefen Einöden, entfernt von dem unruhigen Tumult des Tages, und einzig von Wißbegier und Liebe zur Eintracht beseelt. Er gedenkt in seiner Einsamkeit mit inniger Herzlichkeit seiner Genossen und seiner Familie. [...] Sein Beruf lehrt ihn unermüdliche Geduld, und läßt nicht $\mathrm{zu}, \mathrm{da}$ sich seine Aufmerksamkeit in unnütze Gedanken zerstreue. Er hat mit einer wunderlichen harten und unbiegsamen Macht zu tun, die nur durch hartnäckigen Fleiß und beständige Wachsamkeit zu überwinden ist ${ }^{14}$.

Reichen die zitierten Diskurse auch in Gefilde jenseits der Fiktionalität hinein, so zeichnen sich andere Teile der Hoffmannschen Falun-Handlung gerade dadurch aus, daß sie sich archaisch-fiktionaler Erzählstrukturen bedienen. Sie lassen sich sogar durchaus als märchenhaft charakterisieren, umso mehr, da das Märchen als Gattung weniger durch seine Figuren als durch seine typische Struktur festgelegt ist ${ }^{15}$ : Der depravierte Held Elis begegnet mit Torbern einer Helferfigur, die ihm den Weg zum Glück nach Falun weist, wo jedoch die Kategorien von Gut und Böse, Glück und Unglück durcheinandergeraten, weil unklar ist, ob unten oder oben die glückliche Zukunft zu suchen ist. So läßt sich auch nicht mehr deutlich unterscheiden, welche Teile der Handlung als märchentypische Proben gelten können; denn der Weg des Märchenhelden ist in der Regel ein Sozialaufstieg oder eine Eheanbahnung. Beides geht bei Hoffmann letztlich in Erfüllung - sowohl im Reich der Bergkönigin als auch in der Gemeinschaft der Bergleute, der die Braut angehört, deren treue Liebe sich am Ende mit fünfzigjähriger Verspätung erfüllt. So ergibt sich jedoch eine - auch strukturelle Ambivalenz, die die Einlinigkeit des Märchens weit hinter sich läßt.

Getreu dem Serapionischen Prinzip, das vor die Ausgestaltung ein innen geschautes Bild setzt, das am Ende dieses Prozesses ins äußere Leben eintritt ${ }^{16}$, wird obendrein bei Hoffmann eine gleichsam subjektive Phantastik geliefert. So bleibt das Handlungsfeld der 'märchenhaften' Bergkönigin vollständig auf zwei Traumsequenzen beschränkt, während sich aber Torbern in die Wahrnehmung Elis' einschleicht. Das geht so weit, daß sein 'Erscheinen' ohne relativierende Erzählereingriffe vonstatten geht ${ }^{17}$. Erst in Falun und nachdem ihm der Alte wiederholt begegnet ist, wird Elis mit der dortigen Sage vertraut gemacht, die eine Identifikation Torberns, der so sehr von seiner Vorstellungswelt Besitz ergriffen hat, ermöglicht ${ }^{18}$. Die Begegnung mit dem Wunderbaren erfolgt bei E.T.A.Hoffmann jedoch keineswegs in der 
selbstverständlichen Unmittelbarkeit des Märchens, sondern immer vermittelt durch die Figurenpsychologie, also durch die personale Perspektivierung des Geschehens. In der vor-freudianischen Darstellung Hoffmanns wird das Unterbewußte zunächst im wesentlichen in die Welt unter Tage verbannt. Diese fungiert zum einen als Auslöser besonders intensiver Vorstellungsbilder und zum anderen als Bildreservoir, aus dem sich die Vorstellung des Helden speist, so daß ihm am Ende unten und oben ineinanderfließen, was zugleich die Grenzen von Leben und Tod brüchig macht. Das Anderswo verliert schließlich seine Verankerung im räumlichen Abseits und wird zur omnipräsenten Bedrohung der (bewußten) Existenz.

\section{Hofmannsthal und das Märchen vom Licht}

In Hugo von Hofmannsthals Bearbeitung des Falun-Stoffes wird der Handlungsverlauf des Dramas ganz auf die Lebensgeschichte des Elis Fröbom beschränkt. Dabei wird nicht nur das Wiederfinden der Leiche ausgespart, sondern auch der Bergsturz, bei dem Fröbom zu Tode kommt. So emanzipiert sich dieser Text einerseits von seinen Vorlagen, indem er auf den eigentlichen Sagenkern verzichtet, andererseits beruft er sich in Titel und Lokalisierung genau auf diesen ortsbezogenen Kern. Doch kommt die Ausgestaltung des geographischen Anderswo im Drama mit weit weniger «Realitätsvokabeln» (Hermann Broch) aus als die Erzählung aus den Serapionsbrüdern. Die Handlung, die Hofmannsthal entwickelt, hätte dieses Ortsbezuges kaum bedurft; wohl aber profitiert sie - vor allem mit Blick auf die psychologische Profilierung der agierenden Figuren - von der symbolischen Aufladung der von Hoffmann gelieferten Vorlage, um sich aber zugleich von dieser Folie zu distanzieren.

Es ist gewiß kein Zufall, daß den Zeitgenossen Freuds die Symbolik der Hoffmannschen Traumwelt inspiriert hat: Das unterirdische Paradies im Schoß der ewigen Weiblichkeit wird bei Hofmannsthal ganz unverschlüsselt zum Mutterleib:

Mir wär

Sehr wohl, könnt ich mich in die dunkle Erde

Einwühlen. Ging es nur, mir sollt es schmecken,

Als kröch ich in den Mutterleib zurück.

Er steht auf, fährt mit den Händen wie staunend an seinem

Leib herab.

Mir löst sichs jetzt, daß dieser hier mein Leib

Nur ein Geköch ist aus lebendigen Erden,

[...] spricht gegen den Boden.

Du tiefes Haus, was streben wir von dir,

[...]

Dem Lügensinn, dem Aug allein gehorchend,

Der uns vorspiegelt, was für ewig uns

Verborgen sollte sein, die bunte Welt,

Die wir doch nie besitzen! (S. 23f.) ${ }^{19}$

Diese Selbstanalyse ist jedoch nur der Auftakt für eine Reise ins Anderswo, die den Traum ins Leben treten läßt. Mit ihrem Realismus, der die Darstellung der Psychopathologie des Elis Fröbom in den Mittelpunkt rückt, läßt sich die FalunErzählung E.T.A.Hoffmanns nur bedingt dem phantastischen Genre zurechnen insofern nämlich, als hier die Trennlinie zwischen Imagination und Geistererscheinung unscharf wird. Hugo von Hofmannsthal wagt einen wesentlichen Schritt weiter, indem er die Figuren der romantischen (Kollektiv-)Imagination zu dramatis personae macht, die 
auch außerhalb der Vorstellungswelt Elis' in Aktion treten. «Das ganze Stück spielt in einer magisch geleiteten Welt. [...] Die Welt der Zauber- und Märchenstücke.

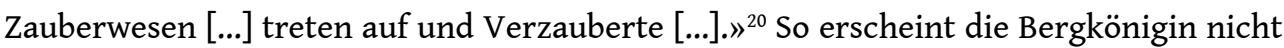
nur als erzähltes Traumbild, sondern als handelnder Gesprächspartner des Helden. Sie begegnet ihm nach einer Verwandlung, die Elis durch intensives Wünschen herbeigeführt hat: «Haus tu Dich auf! gib deine Schwelle her/ Ein Sohn pocht an! auf tu dich, tiefe Kammer» (S.24), ruft er, woraufhin Torbern neben ihm steht, der die Ernsthaftigkeit seines Wunsches zu prüfen hat, bevor sich der Boden auftut und er ins Innere des Berges versinkt. Die Bergkönigin, die er erblickt, negiert ganz explizit seine selbstberuhigende Vermutung, er träume: «Nein, Elis Fröbom,/ Nun träumst du nicht» (S. 29). Am Ende des Gesprächs unter der Erde drängt Elis noch einmal auf eine Erklärung dieses Herabsinkens:

ELIS. Wie konnt ich kommen?

KÖNIGIN. Fragst du aufs neu? Weil du ein Geist wie ich.

Dein Mund sprach mächtige Worte aus.

ELIS. Doch wann?

KÖNIGIN. Du sehntest dich herab, den Boden schlug

Dein Fuß, unwillig trugst du, zornig atmend,

Den Druck der irdischen Luft, dein Blick durchdrang

Die Niedrigkeit, dein Mund verschmähte sie,

Ein ungeheurer Strahl entglomm dem Aug,

Und das Gewürme floh, die Finsternis

Trat hinter sich, so wie sies tut vor mir!

ELIS. Wie kam es über mich!

KÖNIGIN. Es schläft in euch.

Doch ahnt ihrs nicht. Du warst zu Tod erstarrt,

Dein Mund verhangen, deine Augen öd.

Da trats in dir empor, und wie im Traum

Griffst du mit Aug und Mund nach Strahlendem,

Gebunden wie ein Kind, und doch ein Zauberer!

Und halb noch dunkel, halb wie Geister leuchtend,

Ergriffs dich, unbewußt herabzusteigen! (S. 34f.)

Seltsam wirkt hier die Einschränkung «unbewußt», so als wäre Elis in dieser Szene Opfer einer Spaltung, ein Teil von ihm ein Geist, soeben aus dem Schlaf erwacht und doch nur mit der Macht über das Unbewußte ausgestattet. Jedenfalls sprechen die Regieanweisungen gegen die Vermutung, hier würde lediglich ein Traum inszeniert, heißt es doch zu Anfang der Szene «Er versinkt völlig» (S. 28) und am Ende «Er taucht aus dem Erdboden empor» (S. 37). Die aus der Schenke tretende Frau Jensen bestätigt seine Abwesenheit mit einem «So kommt Ihr wieder?» (ebd.). Der Traum wird hier schließlich zur Metapher, die das Wunderbare veranschaulicht, das sichtbar ist und zugleich unsichtbar von Elis Besitz ergriffen hat. Die Bergkönigin zeigt sich aber nur ihm - und dem Zuschauer.

Torbern wird indessen nicht nur von Elis gesehen und gehört, sondern auch von anderen handelnden Personen. Frau Jensen sieht in ihm bei seinem ersten Auftritt einen Bettler (vgl. ebd.), das Kind Rigitze identifiziert ihn sofort, als er in Falun an Dahlsjös Haus vorübergeht, und bringt seine Gestalt mit den Volkserzählungen in Verbindung, die unter den Bergleuten über ihn kursieren und die natürlich auch Kindern erzählt werden, damit sie nicht zu zutraulich gegenüber Fremden sind.

KIND von Angst geschüttelt. Der Torbern!

Der alte Torbern! Ich hab ihn gesehn! 
Verbirgt den Kopf.

ANNA küßt sie. Wer hat dir denn von dem erzählt?

KIND. Der Peer,

Und auch der Onkel, Anna!

ANNA nimmt sie in den Arm. Kind, mein Kind:

Der alte Torbern lebt ja längst nicht mehr!

KIND. Ja, Ja! Er ist ein Zauberer! Der war's!

ANNA. Warum muß er's

gewesen sein?

KIND. Er hat so ausgeschaut.

ANNA. Wie denn?

KIND. Mit blutigen Augen, und der Hals

So lang, und nackt!

ANNA. Rigitze, jetzt merk auf:

Der Torbern war einmal ein weiser Bergmann,

Den hat der Berg verschüttet, und das ist

Zweihundert Jahr jetzt her. (S. 50)

Was in dieser Situation noch rationalisierbar ist und dem Bereich der Fiktion zugewiesen wird, muß gegen Ende des Stückes jedoch der Einsicht weichen, daß Geister unter den Lebenden wandeln: «Es ist nicht bloß in mir», weiß Elis,

Gemeinschaft hat's mit Anderem, das draußen!

Ist eine Welt wie eure, stärker, größer;

die Sterne sind ihr untertan, die Zeiten.

Zu der gehör' ich. (S. 69)

Vor diesem Hintergrund wird denn auch die Verortung Torberns im Märchen hinfällig. Als er erscheint, um Elis zu signalisieren, daß seine Zeit auf Erden abgelaufen ist, ist Anna, seine Braut, Zeuge der Begegnung:

Ich [...] hielt es für ein Märchen.

Nun sah ich ihn, den seit zweihundert Jahren

Kein Auge sah, und sah, wie er zu dir

So redet, wie der Gleiche zu dem Gleichen. (S. 75)

Torbern ist, wie Elis und Anna selbst, Teil der erzählten Welt, die in ihrer Phantastik auch von den Figuren metaphorisch als märchenhaft charakterisiert wird. Elis ist in dieser Version der Falun-Geschichte der Nachfolger Torberns an der Seite der Bergkönigin. Und wenn schon Torberns Lebensgeschichte zur Volkserzählung avanciert, dann hat auch die Geschichte von der gefreiten und verlassenen Braut das Zeug dazu, in die Erzähltradition der Bergleute Eingang zu finden:

Merkt auf: die Hochzeit, die Ihr da ausrichtet

für Euer Kind und mich, den Elis Fröbom,

die wird ein Märlein, das nach hundert Jahren

die Mägde sich erzählen, wenn es dunkelt. (S. 77)

Der Gebrauch des Märchen-Begriffes ist in den genannten Kontexten natürlich eher unreflektiert und nicht poetologisch motiviert. Gemeint ist 'Lügenmärchen', eben die Unwahrheit, Fiktion. Und wer auf der Suche nach gattungsspezifischen Elementen in Hofmannsthals Bergwerk nach strukturellen Eigenheiten einer Märchenhandlung sucht, der findet zwar Rudimente, wie sie auch bei Hoffmann gegeben waren, aber auch einen Schluß, der die Glückssuche des Helden pervertiert: Durch den Wegfall von Bergsturz und Leichenfund fehlt dem Handlungsverlauf jede Versöhnlichkeit. Irdisches Glück ist dem Helden nicht einmal als Leiche beschert, die eine Liebende in ihre Arme schließt. Vielmehr macht er in faustischer Manier seinen Kontrakt mit der Übernatur, schon 
bevor er der irdischen Liebe teilhaftig werden kann. Als ein Lebendig-Toter gelangt er nach Falun, und als Lebendig-Toter verläßt er wiederum das Haus seiner Braut. Erfüllung wird ihm unter der Erde im Schoß der Bergkönigin. Hier mündet der Handlungsverlauf nach Glücksversprechen (die Bergkönigin gesteht ihm ihre Liebe und stellt ihm Teilhabe an der Ewigkeit in Aussicht. «Komm bald!» (S. 37) ruft sie ihm zum Abschied nach der ersten Begegnung nach), Verleihung von Zauberkräften (mit denen er plötzlich zum Bergkundigen wird, der die Geschicke der Firma Dalsjöh umsichtig lenkt: «Habt Ihr's Euch nie geträumt, daß irgendwie/ ein Preis dafür gezahlt müßt' worden sein?», S. 67) und dem erfolgreichen Bestehen von Proben (sämtlichen Versuchen einer sozialen Reintegration - in die Gemeinschaft der Bergleute, in die Familie Pehrson Dalsjöhs und in die Ehe mit Anna - widersteht er).

In seiner Bedeutung für die Textkonstitution des Hofmannsthalschen Dramas wird das Märchen als Gattung bei weitem übertroffen durch die Bezugnahme auf einen einzelnen Märchentext, Das blaue Licht der Brüder Grimm. Eine Anspielung auf dieses Märchen bereitet die Ankunft Elis' in Falun vor, noch bevor die Bergmannssage von Torbern erzählt wird:

KIND. So erzähl mir!

[...] Von der Königstochter.

[...] Die

Hat gehen müssen und dem fremden Mann. -

ANNA. Du weißt's ja so.

KINDwichtig. Der Mann war ein Soldat!

ANNA. Was hat sie müssen?

KIND. Ihn bedienen, alles:

Stiefel ausziehen, Zimmer kehren. [...]

KIND. Warum hat sie ihm dienen müssen?

ANNA. Weißts ja!

KIND. Weil er die Lampe angezündet hat?

Ja? Sag!

ANNA. Die Lampe, freilich.

KIND. Von der Hexe die?

ANNA [...] Da zog es sie, sie mußte hin zu ihm

Und hatte beide Augen halb geschlossen

Und wußte nichts von sich und diente ihm. (S. 48)

Das alludierte Märchen freilich setzt andere Schwerpunkte. In ihm spielt die Königstochter nur eine Nebenrolle. Im Mittelpunkt steht vielmehr der Konflikt zwischen einem König, der seinen Soldaten ohne Rente entläßt und dem Soldaten, der sich für diese Ungerechtigkeit rächen möchte. Dazu dient ihm als Wundermittel ein blaues Licht, das ihm zufällig in die Hände fällt. Der Geist, der erscheint, wenn der Soldat seine Pfeife mit dem Licht entzündet, verhilft ihm zu seiner Genugtuung, die nicht nur die Demütigung der Königstochter, stellvertretend für ihren Vater, sondern auch noch die Erlangung der Königswürde und die Eheschließung mit der Prinzessin umfaßt. Diese jedoch ist stets Objekt der Handlung, zunächst ein Opfer der Rachegelüste und später ein Opfer, das der König bringt, um seine eigene Haut zu retten $^{21}$. Für die Bergwerks-Handlung enthält indessen schon diese kurze Zusammenfassung eine Reihe blinder Motive; denn der oben zitierte Gesprächsausschnitt läßt mit Blick auf Hofmannsthals Drama vor allem zwei Parallelisierungen zu: 
einen findet sich Anna in der Objekt-Rolle angesichts der Macht der Liebe, die sie zu Elis zieht wie ein «blaues Feuer [...], das sie schnell verzehrt» (S. 81). So lautet sogar noch ihr Schlußsatz nach dem Verschwinden Elis': «[E]r steht drinnen/ Im Finstern und er funkelt wie ein Licht:/ Rührt er mich an, so werd ich wieder warm!» (S. 84). Doch ist ja auch Elis seinerseits Objekt der Begierde einer höheren Macht, die ihn an sich zieht, er selbst also auch in der Rolle der Königstochter aus dem Märchen. Und wenn man die Macht über andere in Einheiten der Lichtintensität mißt, dann übertrifft die Bergkönigin, deren Strahlen Elis zu Boden steckt, alles Sichtbare.

Das Märchen vom blauen Licht eröffnet dem Text Hofmannsthals darüber hinaus weitere Prätexte der Romantik und weist damit den Weg in ein rhizomatisches Anderswo der Intertextualität. So begegnet man hier auch Motiven aus Heinrich von Ofterdingens Traum von der blauen Blume, der sich seinerseits mit dem Traum des Elis Fröbom aus E.T.A. Hoffmanns Bergwerken verbindet:

Hinter der Wiese erhob sich eine hohe Klippe, an deren Fuß er eine Öffnung erblickte, die der Anfang eines in den Felsen gehauenen Ganges zu sein schien. Der Gang führte ihn gemächlich eine Zeitlang eben fort, bis zu einer großen Weitung, aus der ihm schon von fern ein helles Licht entgegenglänzte. Wie er hereintrat, ward er einen mächtigen Strahl gewahr, der wie aus einem Springquell bis an die Decke des Gewölbes stieg, und oben in unzählige Funken zerstäubte, die sich unten in einem großen Becken sammelten; der Strahl glänzte wie entzündetes Gold ; nicht das mindeste Geräusch war zu hören, eine heilige Stille umgab das herrliche Schauspiel. Er näherte sich dem Becken, das mit unendlichen Farben wogte und zitterte. Die Wände der Höhle waren mit dieser Flüssigkeit überzogen, die nicht heiß, sondern kühl war, und an den Wänden nur ein mattes, bläuliches Licht von sich warf. [...] Dunkelblaue Felsen mit bunten Adern erhoben sich in einiger Entfernung; das Tageslicht, das ihn umgab, war heller und milder als das gewöhnliche, der Himmel war schwarzblau und völlig rein. Was ihn aber mit voller Macht anzog, war eine hohe lichtblaue Blume, die zunächst an der Quelle stand, und ihn mit ihren breiten, glänzenden Blättern berührte ${ }^{22}$.

Der Weg zur blauen Blume führt durchs Licht. Hier aber zeigt sich die künstlerische Welterkenntnis selbst. Und der Traum wird zum Medium zwischen dem NaturhaftUrsprünglichen und der messianischen Berufung des Individuums, das durch ihn in die Tiefen seiner Seele geführt wird und dort eine ideale Einheit mit dem Universum erfährt $^{23}$. So verbirgt sich hinter dem Licht das Absolute, zu dem Elis nicht durchdringen kann und geblendet wird, solange er der irdischen Welt angehört - ob es sich nun in die Gestalt einer blauen Blume kleidet oder hinter dem Schleier der Bergkönigin verbirgt. So ist es sicher auch kein Zufall, daß diese Figur mit ihren (bräutlichen) Attributen an das von Schiller und Novalis gleichermaßen besungene Bildnis zu Saïs erinnert, das die Wahrheit hinter einem Schleier verbirgt. Wenn man hier in Rechnung stellt, daß sowohl das Gesicht der blauen Blume als auch das der verschleierten Jungfrau in Novalis' Märchen Hyazinth und Rosenblüte aus dem Fragment Die Lehrlinge zu Saïs den Betrachter letztlich auf sich selbst zurückverweisen, kann man erahnen, was Elis unter dem Schleier der Königin erschaut.

ELIs. So darf ich hingehn und dein Antlitz sehn?

KÖNIGIN. Tritt her!

ELIStritt zu ihr.

KöNIGIN steigt die Stufen herab, ihm entgegen, hebt mit der Linken den Schleier von ihrem Antlitz, so daß sein Gesicht, von unten ihr entgegengehoben, ganz von ihrem Abglanz überflutet wird. (S. 35) 
"Abglanz» deutet auf ein Spiegelungsverhältnis hin, das die Verwandtschaft zwischen der Königin und dem «Zauberer» Elis betont, wie sie selbst dies mehrfach tut. Er sieht sich selbst oder jenen Teil seiner selbst, der ihm (noch) unbewußt ist («Keiner wird, was er nicht ist», S. 27), und er sieht seine Zukunft als Zauberer («Gebunden wie ein Kind, und doch ein Zauberer!», S. 35) und Geist («Weil du ein Geist wie ich.», S. 34), eine Zukunft, die zunächst mit einer unterirdischen Hochzeit beginnt und die der Bräutigam in Erinnerung an die erste Begegnung mit der Königin so beschreibt:

[W]ie die den Schleier aufhebt, schwinden ihm

die Sinne, fremd wird ihm sein eigner Leib

und strahlend wie der neugeborne Tag! (S. 77)

Es ist das Licht der Königin, das den Bräutigam entzündet und ihm zu einem neuen Dasein verhilft. Daß diese Verbindung nicht von ewiger Dauer ist, wird in der Stoffgeschichte durch das Auffinden der konservierten Bergmanns-Leiche angedeutet, in Hofmannsthals Drama durch die Geschichte des alten Torbern, der, wiewohl ein Geist und mehrere Jahrhunderte alt, doch sterben muß, um seinem Nachfolger Platz zu machen. Und auch dem Märchen vom blauen Licht fehlt eine Apotheose. Das Licht verleiht zwar Macht, aber nicht Unsterblichkeit. Schließlich weist auch die Ausgangssituation des Märchens in eine ähnliche Richtung: Der König entläßt den Soldaten, als er keine Verwendung mehr für ihn hat, so wie die Bergkönigin sich auch von ihren Dienern nach Belieben trennt.

Das Licht ist zweifellos die zentralste Metapher des Dramas. Als wärmendes Licht steht es - im Kontrast zur (anderen) Welt unter Tage - für die Welt über der Erde, in der den Bergmann die menschliche, körperliche Liebe erwartet. Doch geht diese Liebe im Bergwerk zu Falun mit Fatalismus einher, umso mehr da ihr Licht bei weitem von einem anderen - kalten - übertroffenen wird, das unter Tage leuchtet und Elis längst entzündet hat. Die Bergkönigin ist gleichsam die Inkarnation dieses Lichts, das Erkenntnis und Wahrheit repräsentiert, in letzter Konsequenz das Absolute, das naturgemäß unerreichbar und damit immer anderswo bleibt. Immer wenn sich Elis diesem Licht nähert, wird er wieder auf Distanz gebracht (vgl. S. 35, 102). Dabei ist von Anfang an klar, daß dieser bereits, «halb wie Geister leuchtend» (S. 35) und darum für Ulla so anziehend, der völligen Erleuchtung harrt, die zugleich mit seinem Entschwinden einhergehen muß und deshalb auch jenseits der erzählten Zeit liegt. In einer erstaunlichen Verkehrung des gewohnten, gleichermaßen durch Religion und Aufklärungs-Philosophie gespeisten Koordinatensystems der Rezipienten wird das metaphysische Leuchten des Absoluten aus den höchsten Höhen nach unten, ins Dunkel des Erdinneren, verbannt. Dadurch wird nicht nur optisch der Kontrast erhöht, sondern auch die Ambivalenz gesteigert, die diese Himmel-Hölle kennzeichnet. Hinter der Dunkelheit wartet das Licht. Das wissen Elis und seine Braut gleichermaßen.

\section{Zum Schluß}

Anderswo - das ist in Falun, das ist drunten, unter der Erde, das ist im Inneren der Figuren, das ist das Phantastische - all dies konstitutive Elemente des narrativen Textes und Bedeutungsträger, die wiederum auf ein Anderes verweisen, nämlich auf vorgängige Texte. Im Verlauf der Stoffgeschichte ist überdies eine Verschiebung zu beobachten: Während sich in der Ortssage gemäß ihrer Gattungskonventionen die metaphysische Dimension und das geographische Anderswo die Waage halten, bringt 
die spezifische Ausprägung des Hoffmannschen Realismus neben den räumlichen und geographischen Aspekten auch ein psychisches Anderswo hervor, das sich mit dem Auftreten ausgeformter Figuren herausbildet. Bei Hofmannsthal tritt die Geographie schließlich an der Textoberfläche in den Hintergrund zugunsten des psychischen und phantastischen Anderswo.

Die fremden Welten anderswo sind aber immer auch Textwelten hinter dem Geschriebenen und Gesagten - bei Hofmannsthal, durch die Akkumulation der Stoffgeschichte und die Hinzuziehung weiterer Quellen, in einem zuvor nicht erreichten Ausmaß. Die intertextuelle Aufladung des Bergwerks zu Falun haucht dem Drama ebenso Leben ein wie ein Bühnenbild oder ein Kostüm. Erst durch die Stoffgeschichte der Falun-Sage wissen wir vom dazumal spektakulären Fund der konservierten Leiche und seinen Konnotationen, das Märchen vom blauen Licht, Novalis' und E.T.A. Hoffmanns Traumdarstellungen runden das Bild ab, das Hofmannsthal von seinen Figuren vermittelt. So wird gerade dieser Text zu einem besonders dichten semantischen Geflecht, dessen Entschlüsselung Lesern und Zuschauern einschlägige Vorkenntnisse abverlangt. Mag sein, daß nicht zuletzt diese Erklärungsbedürftigkeit seine Popularität verhindert hat. Der Aufmerksamkeit weiterer Forschungsarbeiten sei er jedoch empfohlen.

\section{NOTES}

1. Vgl. dazu Bernd Hamacher: "Aspekte der Dramenanalyse", in: Thomas Eicher, Volker Wiemann (Hg.): Arbeitsbuch: Literaturwissenschaft, 3, vollständig überarbeitete Auflage 2001, Paderborn, Schöningh (UTB, Große Reihe), 1996, S. 133-170; $137 f$.

2. Vgl. Curt von Faber du Faur: «Der Abstieg in den Berg. Zu Hofmannsthals Bergwerk zu Falun », Monatshefte XLIII, 1951, S. 1-14; S. 5.

3. Vgl. dazu Thomas Eicher (Hg.): Das Bergwerk von Falun. Varianten eines literarischen Stoffes, Münster, LIT Verlag, 1996, S. $209 f$.

4. Vgl. Gotthilf Heinrich Schubert: Ansichten von der Nachtseite der Naturwissenschaft, Dresden, Arnoldische Buchhandlung, 1808, S. 215f.

5. Vgl. Eicher (Hg.): Das Bergwerk von Falun, a.a.O. S. 13-206.

6. Vgl. dazu Helmuth Mojem: «Hoffnungsfroher Widerstand. Johann Peter Hebels Unverhofftes Wiedersehen zwischen Idylle und Utopie», Zeitschrift für deutsche Philologie 111, 1992, S. 181-200.

7. Vgl. Gerhard Heilfurth: Bergbau und Bergmann in der deutschsprachigen Sagenüberlieferung Mitteleuropas, Bd. 1 - Quellen, Marburg, N. G. Elwert, 1967, S. 531 .

8. Vgl. Karlheinz Stierle: «Die Struktur narrativer Texte. Am Beispiel von Johann Peter Hebels Kalendergeschichte Unverhofftes Wiedersehen», in: Helmut Brackert, Eberhard Lämmert(Hg.): Funk-Kolleg Literatur, Bd. 1, Frankfurt a.M., 1977, S. 210-233; S. $222 f$.

9. Ernst Theodor Amadeus Hoffmann: Die Bergwerke zu Falun, in: ders.: Die Serapionsbrüder, München, Winkler, 1963, S. 171-197; S. 188.

10. Vgl. dazu Iris Denneler: Die Kehrseite der Vernunft. Zur Widersetzlichkeit der Literatur in Spätaufklärung und Romantik, München, Fink, 1996, S. 167-169. 
11. Vgl. Klaus Detering: Die Poetik der inneren und äußeren Welt bei E.T.A. Hoffmann. Zur Konstitution des Poetischen in den Werken und Selbstzeugnissen, Frankfurt a.M. u.a., 1991, S. 256-285.

12. Vgl. Eicher (Hg.): Das Bergwerk von Falun, a.a.O., S. $211 \mathrm{f}$.

13. Hoffmann: Die Serapionsbrüder, a.a.o., S. 197.

14. Novalis (d.i. Friedrich von Hardenberg): Heinrich von Ofterdingen, in: ders.: Werke, Tagebücher und Briefe Friedrich von Hardenbergs, hg. von Hans-Joachim Mähl und Richard Samuel, München/ Wien, Hanser, 1978, S. 237-413; S. 291.

15. Vgl. dazu Thomas Eicher: «Einleitung», in: Märchen und Moderne. Fallbeispiele einer intertextuellen Relation, hg. von Thomas Eicher, Münster, LIT-Verlag, 1996, S. 7-20, 8-10.

16. Hoffmann: Die Serapionsbrüder, a.a.O., S. 55.

17. Während es anfangs noch heißt: «Elis glaubte den alten Bergmann erkannt zu haben» (Hoffmann: Die Bergwerke zu Falun, a.a.O., S. 180), sieht er ihn auf seinem Weg nach Falun «wie er aus einer Schlucht, aus dickem Gestripp, aus dunklem Gestein plötzlich hervor[tritt]» (ebd.). «Der Alte stand vor ihm riesengroß» (ebd.). Später, als er unter Tage arbeitet, sieht er «dicht neben sich einen schwarzen Schatten und erkannte [...] den alten Bergmann von Götaborg» (ebd., S. 187).

18. Vgl. ebd., S. $188 f$.

19. Hier und im folgenden beziehen sich die eingeklammerten Seitenzahlen auf Hugo von Hofmannsthal: Das Bergwerk zu Falun, in: ders.: Dramen 4, hg. von Hans-Georg Dewitz, Frankfurt a.M., S. Fischer, 1995 (= ders.: Sämtliche Werke, Kritische Ausgabe, Bd.6), S.9-103. Regieanweisungen werden der Übersichtlichkeit halber kursiviert.

20. Faber du Faur: «Der Abstieg in den Berg», a.a.O., S. 6.

21. Vgl. Eicher (Hg.): Das Bergwerk von Falun, a.a.O., S. 215-217.

22. Novalis : Heinrich von Ofterdingen, a.a.O., S. 242.

23. Zum universellen Charakter des Symbols vgl. auch Hans Jürgen Balmes: Novalis-Kommentar, München, 1987 (= Novalis: Werke, Tagebücher und Briefe Friedrich von Hardenbergs, hg. von HansJoachim Mähl und Richard Samuel, Bd. 3), S. 158f.

\section{RÉSUMÉS}

Das Anderswo, dem dieser Aufsatz nachgeht, liegt in Texten, die durch intertextuelle Verweise heraufbeschworen werden, um der Darstellung eine zusätzliche Dimension zu verleihen. Hofmannsthals Drama nimmt im Titel wie im Handlungsverlauf Bezug auf eine ganze Stofftradition, der sich vor allem die Romantik angenommen hat. E.T.A. Hoffmanns Version gibt die Folie ab, auf der sich das Stück entwickelt. Hofmannsthals Modifikationen jedoch sind entscheidend. So verzichtet er am Ende auf den Sagenkern der Erzählung Hoffmanns, eben die Verschüttung eines Bergmanns, der fünfzig Jahre nach seinem Tod als konservierte Leiche geborgen und von seiner treuen Braut wiedererkannt wird. Unter Verzicht auf diesen Abschluß wird bei Hofmannsthal der Blick auf die Psychologie der Figuren gerichtet - so will es zumindest die Textoberfläche. Doch leben auch um 1900 die romantischen Adaptionen des Stoffes als Zitate fort und werden überdies durch andere Anspielungen auf Texte der Romantik - etwa von den Brüdern Grimm und Novalis - ergänzt. Da Hofmannsthal obendrein die Inszenierung der Psyche durch phantastische Elemente wie Geisterspuk und Zauberei betreibt, verstärkt sich die Märchenhaftigkeit des Dramas. 
L'ailleurs que cet article explore réside dans les écrits que l'intertextualité convoque pour conférer à la représentation une dimension supplémentaire. Dans La Mine de Falun de Hofmannsthal, titre et déroulement de l'action se réfèrent à un sujet riche de toute une tradition dont les principaux représentants sont les romantiques. C'est la version de Hoffmann qui fournit à la pièce sa toile de fond, mais les modifications opérées par Hofmannsthal sont décisives. Il renonce ainsi à la fin au cœur de la légende, encore présent chez Hoffmann : la conservation, cinquante ans après sa mort, du corps d'un mineur enseveli sous un éboulement, et son identification par sa fiancée qui lui a gardé fidélité. L'abandon de ce dénouement place la psychologie des personnages au premier plan - c'est du moins la réalité textuelle manifeste. Mais, même aux alentours de 1900, les adaptations romantiques du sujet poursuivent leur vie sous forme de citation, complétées par des allusions à d'autres textes du romantisme, tels ceux des frères Grimm ou de Novalis. En outre, Hofmannsthal fait intervenir des éléments fantastiques - esprits et sortilèges - dans la mise en scène de la psyché, ce qui accroît le caractère féérique du drame.

The elsewhere this article consideres lies in texts that the inter-textuality summons to give the representation a supplementary dimension. In The Mines of Falun, title and plot refer to a subject rich of a whole tradition whose main representatives are the Romantics. It is Hoffmann's version that gives the play its backcloth but the modifications made by Hofmannstahl are decisive. He renounces thus eventually to the heart of the legend, still present in Hoffmann: the conservation, fifty years after his death, of the body of a miner who has been buried under a mass of fallen earth and its identification by his bride who remained faithful to him. The abandon of this denouement puts characters' psychology in the foreground - it is at least the manifest textual reality. However, even around 1900, the Romantic adaptations of the subject make their way as quotations, completed by allusions to other texts from the Romantic era, such as the ones of the Brothers Grimm or Novalis. In addition, Hofmannsthal brings into play fantasy elements - spirits and spells- in the staging of the psyche, which increases the drama's fairy character.

\section{INDEX}

Mots-clés : E.T.A. Hoffmann, Novalis, Grimm, contes romantiques, intertextualité oeuvrecitee Das Bergwerk zu Falun, Die Bergwerke zu Falun, Die Serapionsbrüder, Heinrich von Ofterdingen, Das blaue Licht

\section{AUTEURS \\ THOMAS EICHER}

Universität Dortmund 